\title{
Urban transformation and sociocultural changes in King Abdullah Economic City (KAEC) 2005-2020: Key research challenges
}

\author{
Oumr Adnan Osra ${ }^{*}$
}

University of Sydney, Sydney, Australia

\author{
Key Words \\ Local identity \\ Sociocultural values \\ Housing \\ Neighbourhood \\ Jeddah \\ KAEC
}

Received: 13 October 2016

Accepted: 27 February 2017

Published: 30 June 2017

\begin{abstract}
The main aim is to explore how local identity is being expressed in contemporary urban development in Saudi Arabia. The paper addresses this aim by reviewing the case study design, which is the most appropriate research method that widely helps in assessing two case studies of urban development in two main cities in Saudi Arabia, namely the traditional city of Jeddah and the new masterplan of KAEC, to identify how Blexible are the key Islamic socio-cultural determinants in terms of designing residential neighborhoods, housing design, and built environment generally to accommodate people from different socio-cultural backgrounds. The outcomes of this study would help stakeholders and other researchers to better understand the basic Islamic determinants in terms of urban development, which do not distinguish between people from different socio-cultural backgrounds when it comes to forming the built environment, as they are mainly focused on achieving public benebits such as maintaining neighbors' rights.
\end{abstract}

(C2017 The Author. Published by TAF Publishing

\section{INTRODUCTION}

Saudi Arabia, in the meantime, has undergone a huge economic transition never before witnessed in its history, except perhaps when oil prices dramatically boomed in 1973 during the famous oil crisis that became a great transitional point in the country's development. Since this crisis, fossil oil has been considered a major source of income for the country. Nowadays, it seems that relying on fossil oil as the country's only source of income has become an ineffective and unstable prospect under the existing circumstances of falling oil prices in the world market, even though the local demand for fossil fuel has dramatically increased ( Lahn and Paul, 2011). As a panacea for the possible economic crises that could affect the country, King Abdullah Bin Abdul-Aziz Al Saud (who passed away on January 23, 2015) announced in 2005 a master plan known as the KAEC in Rabigh on the Red Sea as one of four such economic cities to be built(Moser et al., 2015). According to Moser et al. (2015), the urban notion of economic cities is basically aimed at attracting private capital and expatriates to invest in Saudi Arabia by offering several economic projects that transcend the oil industry, thereby transferring Saudi Arabia to 'a nerve center of global business' (Moser et al., 2015). The Saudi government enthusiastically plotted a path to become a global hub for the industry, R\&D, education, and tourism (Moser et al., 2015). Shifting

\footnotetext{
${ }^{*}$ Corresponding author: Oumr Adnan Osra

†Email: oosr3429@uni.sydney.edu.au
} 
toward a post-oil era seems a great solution in the meantime, as it would definitely contribute to tackling existing economic issues due to dramatic decrease in oil prices coupled with a rising local demand for fossil oil, such that achieving the National Transformation Plan in Saudi Arabia has become a patriotic issue of survival (Moser et al., 2015). However, this shift could be difficult to sustain by employing an inexperienced local workforce, as expatriates would prefer to keep their own sociocultural lifestyle which could lead to social tensions between Saudis and expatriates as well as between Saudis themselves. On the one hand, some Saudis welcome living side by side with non-Saudis, as they realize that accepting that situation would mean changing some of their sociocultural values and norms, and so they do not have any concerns about such co-existence. On the other hand, many Saudis are strong adherents of Islamic sociocultural values and norms which could become a sociocultural barrier when dealing with non-Saudis, especially non-Muslims. As a result, various radical and incremental changes and innovations (physical, economic, educational, societal, and cultural) have been introduced by the Saudi government under the slogan: "modernization without westernization." The slogan highlights the government's efforts to encourage an incremental change towards integrating development and modernization with traditional cultural values (Christiansen et al., 2013). The KAEC in Rabigh, which will be discussed at length as a case study in this paper in addition to the built environment in Jeddah city, will help in exploring how the local identity is being expressed in contemporary urban development.

\section{Objective}

The aims of this study were to develop the scholarly English oral presentation ability of the pre-service teachers majoring in English, studying at Faculty of Education, Suan Sunandha Rajabhat University, using Miller's model and to investigate their attitudes towards teaching based on this model.

\section{Main Aim}

The main aim is to explore how the concept of local identity is being expressed in contemporary urban development in Saudi Arabia. The paper addresses this aim by reviewing the case study design, which is the most appropriate research method that widely help in assessing two case studies of urban development in two main cities at Saudi Arabia, namely the traditional city of Jeddah and the new masterplan of KAEC, to identify how flexible are the key Islamic sociocultural determinants in terms of designing residential neighbourhoods, housing design, and built environment generally to accommodate people from different sociocultural backgrounds.

\section{LITERATURE REVIEW}

In the majority of architectural works, residents tend to somehow ensure continuity in their sociocultural values by using architectural languages to produce unique forms that reflect their sociocultural experiences from the past (Ayalp, 2012). These experiences are sociocultural practices that are mostly governed by societal principles. The sense of continuity will be realised when sociocultural values are converted to traditions under the aim of bridging the past with the present (Albarqawi, 2012b). Tradition has the ability to build up sociocultural practices through the use of architectural languages in building communities (Al-Hathloul and Muhammad, 1999). Tradition works as a measuring tool when people start to deal with others who hail from other sociocultural backgrounds (Al-Hathloul and Muhammad, 1999). As a result, adopting the process of sociocultural values from other cultures will be tested through the aforementioned measuring tool in order to make 
the appropriate decision and eliminate any sense of alienation when dealing with people from other sociocultural backgrounds (Albarqawi, 2012b). However, there is a clear lack of clarity about how adherence to sociocultural values and norms can impede urban development in KAEC. On the one hand, the majority of Saudis are strong adherents of Islamic sociocultural values, while on the other hand, expatriates are also adherents of their own sociocultural values, so how KAEC will be able to handle those different if not opposing sociocultural values and norms is something that ought to be identified. Also to be identified is how flexible are the key Islamic sociocultural determinants that shape both residential neighborhood patterns as well as housing units so as to accommodate both Saudis and expatriates.

By looking at the government's slogan "modernization and non westernization", the Saudi government clearly realizes that constructing KAEC should embed certain changes to sociocultural values. However, the government as well as stakeholders do not clarify what kind of changes could come about in terms of sociocultural values and norms, and the extent to which those values and norms are flexible enough to be adopted. Some thinkers assume that westernization is generally the same as modernization. However, in terms of KAEC, how westernization and modernization can be defined remains open to question. By looking at the master plan of KAEC, it is difficult to know if this project is meant to be built in Saudi Arabia or somewhere else. This issue has been discussed at length in the earlier literature by Gharipour (2015). Gharipour (2015) remarks that the majority of countries in the Middle East are suffering from a lack of association between the building designs and local cultures. Gharipour (2015) extended the idea to show how the strong conflict between diversity of sociocultural values and building designs has resulted in non-homogenized architecture in the Middle East as well as the loss of local identity in the urban spaces (Aly, 2011). However, some of the early literature had focused on sociocultural changes from a positive perspective (Moser et al., 2015; Howard, 1946). Moser et al. (2015) asserted that the construction of KAEC represents a leap from conservative sociocultural values and norms towards modernity by adopting the entrepreneurial urban approach.

The paper covered specific gaps in the earlier literature about KAEC such as discussing the difficulties of governance and how they might affect the future of the project. Then, offering a preliminary overview as well as an analysis of the KAEC master plan, it finally exposes local and global ambitions in the construction of KAEC (Moser et al., 2015). To some extent, I personally agree with looking at the issue from a more positive perspective. However, previous literature do not deal with how the urban approach and features of the city can make assessing the sociocultural life possible, as the city is basically designed to offer healthy living and economic wellbeing. This is related to the idea of the Garden City, where the urban pattern of the city was designed to maximize healthy living and economic wellbeing (Howard, 1946). Howard (1946) discussed the matter of urban development from another perspective, wherein he focused on studying the overlapping relationships in the urban functions within the community to develop a relationship between the city urban fabric on the one hand and sociocultural, political, and economic matters on the other (Howard, 1946). There is a significant relationship between people and the buildings in which they live, which express sociocultural values through the use of architectural elements as a communication language. Identity, then, may be made manifest in patterns of thought driven by principles according to a specific culture (Al-Naim, 2008). This formation is described as a long-term process, which eventually constructs architectural symbols that embody meaning and reflect the values of that particular society (Albarqawi, 
2012b). On the one hand, some early literature investigated people's perceptions about changes in the urban environment as a mode of inquiry that can determine the correlation between urban transformation and people's perceptions about their identity (Albarqawi, 2012b; Al-Naim, 2008). Also, discussed how urban development in Putrajaya came about when conservatism arose within the Muslim community in Malaysia (Moser, 2012). She remarked that instead of dealing with the diverse population from a wide variety of sociocultural backgrounds in order to develop a unique urban fabric, the state decided to apply "a fantasy Middle Eastern style for secular national buildings" (Moser, 2012). In the same paper, Sara tried to investigate the architectural development in Putrajaya as a manifestation of social and religious trends, and she discussed how urban development in that city can be improved in order to homogenize with existing sociocultural patterns in Malaysia (Moser, 2012). On the same matter, Masad (2008) mentioned that Dubai consists of several segregated communities due to differentiation in some significant aspects, which should be taken seriously by the government in order to avoid any tensions between locals and expatriates: "...The social architecture of Dubai is premised on a sharp division and separation of the main three communities: local Emiratis, western, Arab and subcontinental expatriates, and South Asian workers.

These communities are generally differentiated by their civic rights, socio-economic status, residential location, lifestyle priorities, and cultural identities. Some of the spheres of separation are the result of the kinds of jobs people have and how much money they earn; others exist by virtue of the natural gravitation of different groups of people towards communities and localities that reflect their national or cultural identity. Consequently, these groups enjoy different sets of choices and freedoms"(Masad, 2008). Elsheshtawy, $(2008,2010)$ added in a report for the UN-ESCWA under the title, The Status of the Arab City, where he discussed how recent urban development in the Middle East especially in the Arabian Gulf region has resulted in a wide rift in terms of sociocultural sustainability because it is based on inequality. On the other hand, Nezar Al-Sayyad pointed out that differentiation, and not homogenization in cultural values, is being seen worldwide where people belong to a variety of cultural backgrounds and identities (Albarqawi, 2012b). Al-Barqawi (2012b) mentioned thus: "It is possible to argue that identity is mentally constructed, either consciously or unconsciously and is physically manifest in patterns of forms that determine cities as a production of a specific culture in relation to the other' "( Albarqawi, 2012a).

\section{King Abdullah Economic City (KAEC)}

The megacity covers around 180 square kilometers with an amazing view of the Red Sea. It is located in Rabigh, at around $133 \mathrm{~km}$ from the holy city of Makkah, $219 \mathrm{~km}$ from Medina, and just $100 \mathrm{~km}$ from the commercial hub, Jeddah ( Shoaib and Ramin, 2015). The strategic location of KAEC has been chosen by the Saudi government to attract investors from all over the world, hoping to achieve the national transition plan through economic diversity and shifting to post-oil, as well as creating one million jobs for Saudi youths by 2035, for according to Glum (2015): “Two-thirds of the nation's 29 million-person population is under age 30". KAEC city consists of six zones, at least at the announcement of the project: industrial district, seaport, residential and resort area, educational zone, central business district, and financial island (Economic Cities Saudi Arabia, 2010; Ernst \& Young, 2015). But ten years after announcing the megacity, just around 15 percent has been constructed at this pop-up metropolis in the middle of the desert (McLelland, 2015). The estimated population for people who move to live there is approximately 50,000 by 2020 , which is supposed to skyrocket to two million by 2035 (Swiss Business Hub GCC, 2010; McLelland, 
2015). EMAAR Properties has stated that the master plan of KAEC was mainly developed to form "unique identities that contribute to a holistic city vision" (KEAC, 2013). However, by looking at the master plan, residential neighborhood plots, and housing units, it is not clear what type of identity this city is trying to create, and how it can provide a sample of built environment that is flexible enough to accommodate people from different sociocultural backgrounds on the one hand, and still express the local identity of the country on the other (Fig. 1\&2).

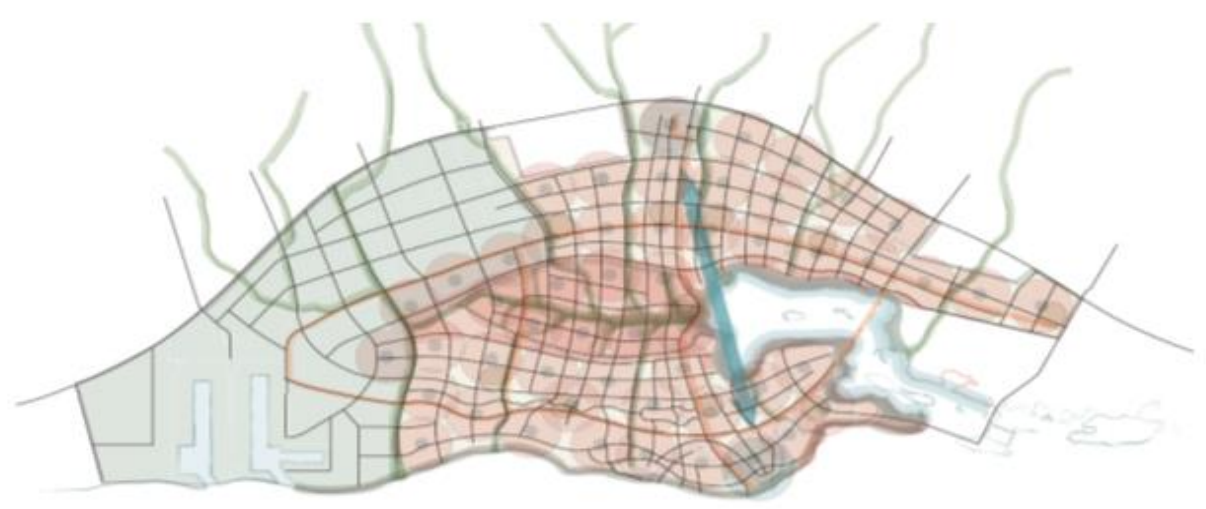

FIGURE 1. Shows a sample of the built environment at one of the residential district centers Source: (KEAC, 2013)

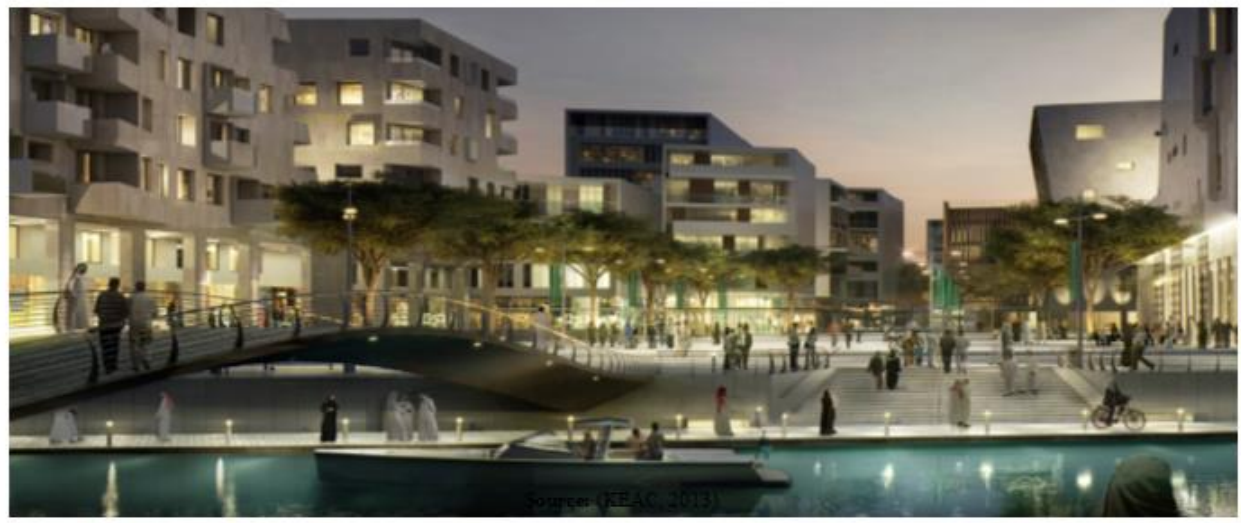

FIGURE 2 . Shows a sample of the built environment at one of the residential district centers

Source: (KEAC, 2013)

\section{Jeddah}

Jeddah is the largest city in the western region of Saudi Arabia. Due to its economic status as a commercial hub during the Saudi regime and even before that, it has come to occupy around 5,460 square kilo meters at present (Saudi Commission for Tourism and National Heritage, 2013). The city's urban sprawl has rapidly increased since the oil boom (from 1938 until the present era), and this continuous expansion has resulted in changing the physical environment, lifestyle and local identity of Jeddah city (Salagoor, 1990). There are other factors that significantly contributed to changing the urban fabric of the city 
such as the introduction of the automobile, construction techniques and building materials. Therefore, the spatial context of the housing unit has also changed, which has resulted in new architectural features and loss of local identity. When talking about the city as a whole, the city was distinguished by the offer of a homogenized urban environment where the local architecture complements the city's compact urban fabric. However, it has transformed into a heterogeneous city where the majority of housing typologies do not communicate the sociocultural values of city residents (Fig. 3). Moreover, the city urban pattern has lost its unique features, which functionally contributes to solving environmental matters and matching the sociocultural values of city residents. For example, the narrow winding streets have been replaced by wide roads, intersections and flyovers, where the public space has been transformed to become a parking area, and so on. Due to this rapid urban transformation, the city has witnessed dramatic changes in its physical environment, which led to a gradual change in the indigenous sociocultural values of its residents. Subsequently, the society itself has lost its traditional local pattern and become "a semi-cosmopolitan environment of the twentieth century"(Harbi, 1989).

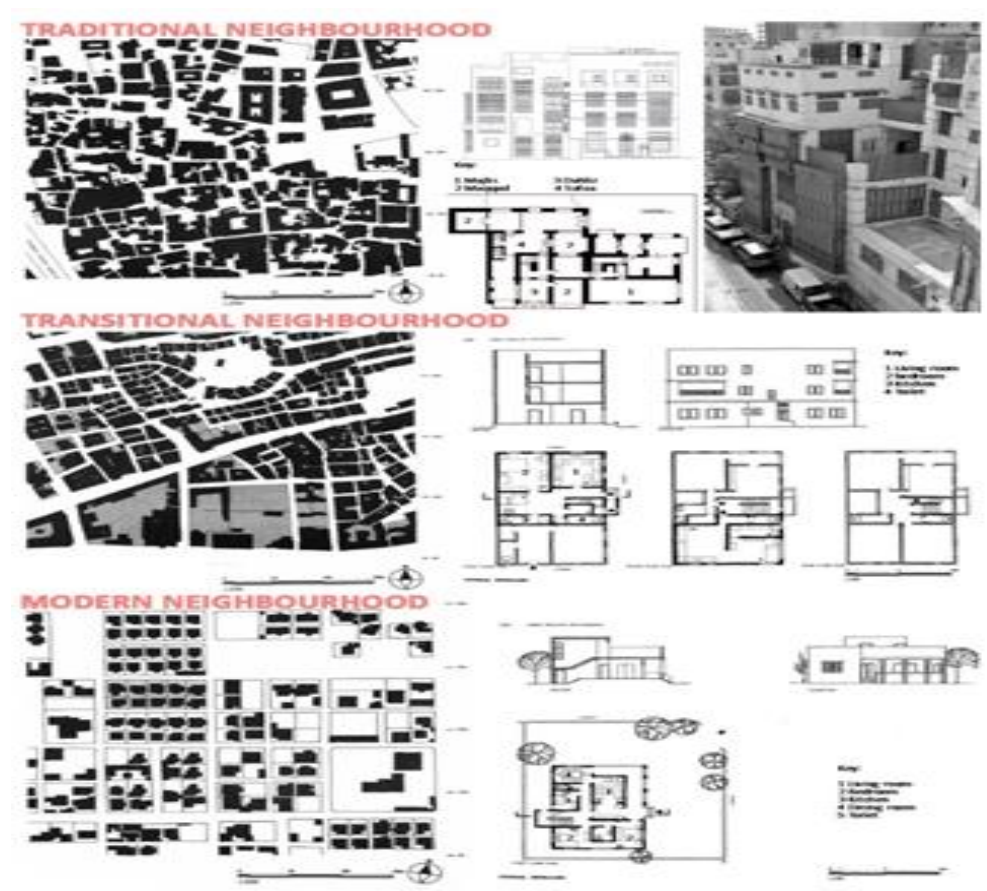

FIGURE 3 . Shows the different stages of the urban morphology on the level of the neighborhood and houses' design at Jeddah Source: Adopted from (Faden, 1977; Saudi Commission for Tourism and National Heritage, 2013)

\section{CASE STUDY DESIGN}

This method has been adopted in several disciplines such as sociology and cultural studies, and researchers have used this design either as an individual approach or combined with other paradigms. The case which is supposed to be assessed could be an individual, organization, event, or action, existing in a specific time and place (Yin, 2009). There are a variety of definitions for the case study research at the literature. Some of them asserted on getting reliable outcomes due to collecting evidences from different resources, while other definitions focused on the aims behind using this design. For example, Yin's (2003) definition is an example of such an all-inclusive descriptive definition: "A case study is an empirical in- 
quiry that investigates a contemporary phenomenon within its real-life context, especially when the boundaries between the object of study and context are not clearly evident. It copes with the technically distinctive situation in which there will be many more variables of interest than data points, and as one result relies on multiple sources of evidence, with data needing to converge in a triangulating fashion, and as another result benefits from the prior development of theoretical propositions to guide data collection and analysis" (Yin, 2003). Yin's (2003) previous definition strongly focused on looking at the case study in its natural context, which is considered the most significant point in the case study research which the researcher cannot manipulate; he also mentioned that the case study design is "a comprehensive research strategy"(Fageha, 2014; Dul and Tony, 2008).

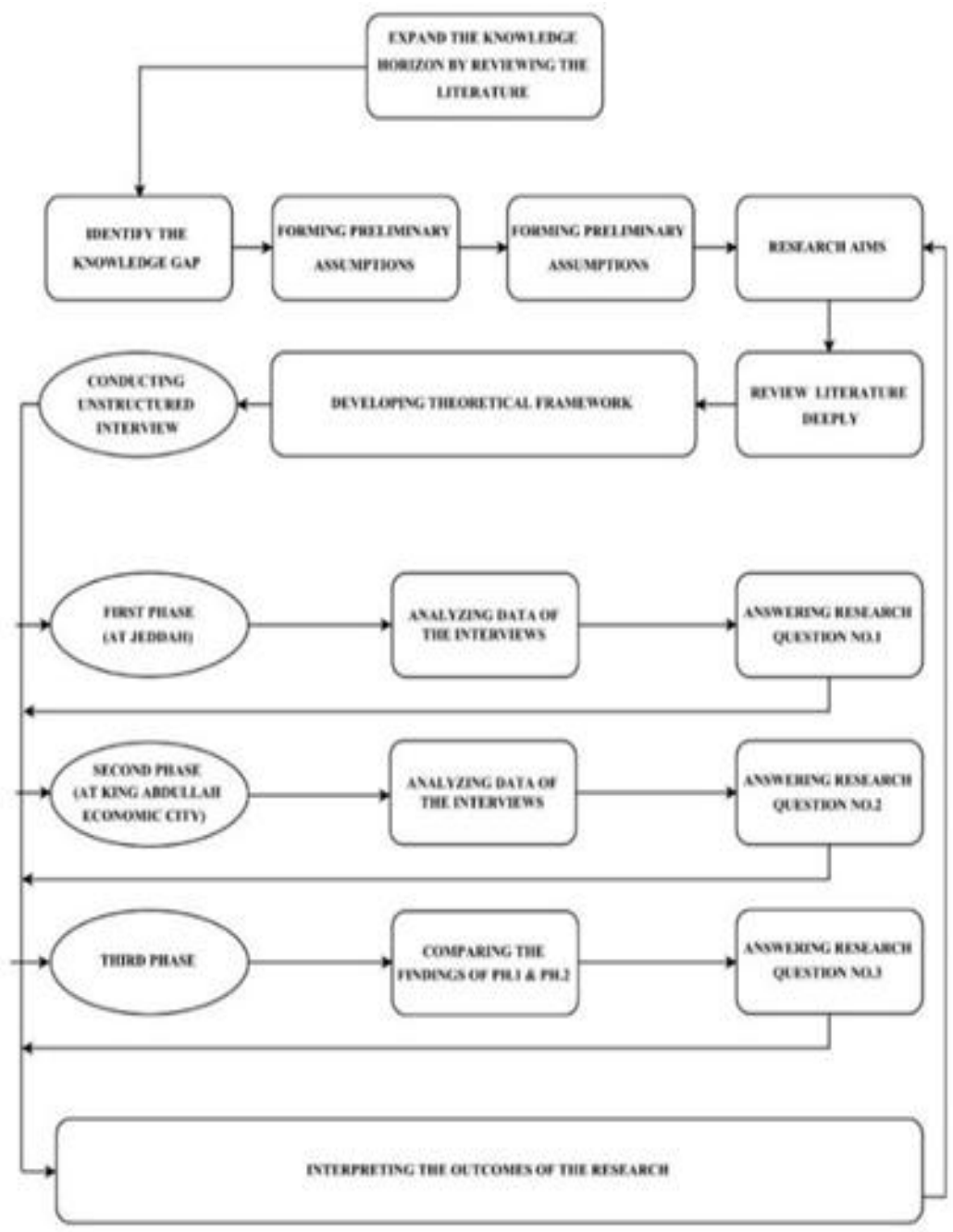

FIGURE 4 . Shows the research stages

Source: Adopted from (Fageha, 2014)

While Dul and Tony (2008) extended the case study's definition to include what Yin (2003) mentioned in terms of studying the case in its natural context, some other aspects have been mentioned in other literature: "A case study is a study in which (a) one case (single case study) or a small number of cases (comparative case study) in their real life context are selected, and (b) scores obtained from these cases are analysed in a qualitative manner" (Dul and Tony, 2008). Yin (2003) has also reckoned that the case study research will 
be effective and useful when the research questions start with "what" or "why" (Yin, 2009). Another significant aspect that could support using that approach is where the researcher cannot manipulate participants' behaviors, when the researcher needs to comprehend the contextual conditions because he thinks that they are related to the studied phenomenon; finally, when the relationship between the phenomenon and contextual conditions is not clear and required further clarification (Baxter and Susan, 2008). This method becomes widely useful when the studied phenomena, which correlate the sociocultural values to the urban patterns in both the city of Jeddah and KAEC, are neither thoroughly studied nor sufficiently theorized. Hancock and Bob (2011) mentioned that there are several features of the case study design. First, this design aims to investigate a phenomenon, organization, or representative of a group; he extended this to include investigating "a particular event, situation, program, or activity" (Hancock and Bob, 2011). Second, he asserted investigating the phenomenon within "its natural context, bounded by space and time". Third, he added that the case study design is mainly descriptive because it depends on collecting evidence from several resources (Hancock and Bob, 2011). Hancock and Bob (2011) added that “...it employs quotes from key participants, anecdotes, narratives composed from original interviews, and other literary techniques to create mental images that bring to life the complexity of the many variables inherent in the phenomenon being studied" (Hancock and Bob, 2011). Meanwhile, Yin (2003) mentioned five significant components for the case study design: the questions of the study, data to be collected, questions' propositions, unit of analysis, and finally the overall design should logically show a framework to connect the collected evidence with propositions and the strategy of analyzing the outcomes (Yin, 2009). In my opinion, the perspectives of both Hancock and Bob (2011) and Yin (2003) complement each other. There are several designs for the case study research, which are different based on three factors: types, characteristics of the design, and disciplines' types. Hancock and Bob (2011) mentioned that there is a significant relationship between the case study design and the disciplinary orientations, and he pointed to Merriam's classification of the case study design. She mentioned four main orientations for the case study research: "ethnographic, historical, physiological, sociological orientations" (Hancock and Bob, 2011). Ethnographic orientation usually helps in understanding the interactions between groups; historical orientation precisely investigates a particular event or program; physiological orientation helps in understanding human behaviors. As a result, the main orientation in this research is sociological. This orientation includes investigating a variety of matters mentioned by Hancock and Bob (2011): "Families, religion, politics, health care demographics, urbanization, and issues related to gender, race, status, and aging. Sociological case study research with its focus on society, social institutions, and social relationships, examines the structure, development, interaction, and collective behaviour of organized groups of individuals" (Hancock and Bob, 2011). Yin (2009) and Stake (1995) have divided case study design into several types. First, they defined three main categories for the case study design: explanatory, exploratory, and descriptive (Baxter and Susan, 2008; Stake, 1995). While Yin (2003) mentioned three main types of case study design (i.e. holistic, single, and multiple case-studies), Stake (1995) mentioned the following three types: instrumental, collective, and intrinsic (Baxter and Susan, 2008; Stake, 1995). In fact, this research adopts the explanatory design, which intends to develop "cause and effect relationships". By applying this design, the research aims to identify the reasons that led to the urban transformation of Saudi Arabia, especially the city of Jeddah and KAEC, to clarify the relationship between the built environment generally and sociocultural values and norms (Hancock and Bob, 2011). According to Yin (2003) the multiple case study design 
is highly pertinent to providing a larger picture of the studied phenomenon or even when the research compares different studies (Baxter and Susan, 2008). This research aims to apply a multiple or collective case study approach that ensures ample opportunities for the researcher to identify the key determinants of the urban fabric in the traditional city of Jeddah and KAEC in relation to the sociocultural discourse; similarities and differences could be identified between the two cases about the local identity, and how it has been expressed in terms of major urban development in Saudi Arabia. One of the important advantages of using this approach is related to having robust and reliable evidence as well as reliable results when applying this design.

\section{Case Study Protocol}

Yin (2009) came up with a useful definition for the case study protocol: "The protocol is a major way of increasing the reliability of case study research and is intended to guide the investigator in carrying out the data colᄀlection from a single case (again, even if the single case is one of several in a multiple-case study)" (Yin, 2009). Therefore, this research, which deals with multiple case studies, should make use of such a case study protocol. Generally, a case study protocol consists of several phases. First, an investigator should offer brief information on the case study, which may be either individual or multiple, and should include literature about the research topic, research objectives, the topic of investigation, and other possible issues (Yin, 2009). Second, an investigator should prepare for the field work by developing a protocol for field procedure, which includes several factors such as having the appropriate credentials, visiting the case studies' sites, identifying the data's sources, and developing ways to help easily identify the information (Yin, 2009). Third, in case study research, investigators should focus on the case study's questions during the stage of collecting the evidence so as to be able to sufficiently address the research questions (Yin, 2009). Finally, investigators should develop a general framework for reporting the case study, which includes clarifying the source of the data that has been collected on the case study and how it will be presented (Yin, 2009). According to Yin (2009) there are several advantages for applying the case study protocol. First, it helps the investigator to focus on the research topic (Yin, 2009). Second, it will help identify the possible audience to be dealt with during the investigation process of the case study-until reporting it (Yin, 2009).

\section{Case study Evidences}

The research will collect the data from multiple resources to alleviate any risks relating to depending on one individual source for collecting the research information. These multiple resources consist of five sources of evidence: documentation, archival records, interviews, direct observation, and participant observation (Hancock and Bob, 2011). There are several benefits for collecting data from multiple resources. One significant benefit is that the research is then able to discuss multiple aspects such as urban development, house typologies, the built environment generally, and sociocultural discourse (which is difficult to achieve by depending on one single resource for the data). Another important benefit is privacy; some people could reject either the process of inspecting the internal spaces of the house by the researcher or answer questions that discuss sensitive matters. Moreover, it can help develop a database for the studied phenomenon. Finally, a researcher should collect a number of evidence to ensure a high quality research, which might also help in ensuring the validity and reliability of the research outcomes (Yin, 2009). 


\section{Source of Evidences}

Documentation: Yin (2003) stated that there are different forms of documentation, which will also be used in this research. Personal documents that include letters, memoranda, and email correspondences (Yin, 2009). Formal studies include studies that have been conducted on the same case study (Yin, 2009). Administrative documents include different kinds of proposals or reports (Yin, 2009). Finally, articles which have been published in newspapers (either printed or online) will also be used in this research (Yin, 2009). Using these documentations has several benefits in terms of corroborating the collected evidence from other resources, confirming the correct name of organizations (which interviewees could mention during the interviews), providing more details about the collected evidence from other resources, and the researcher can use documents for inferences (Yin, 2009). In case study research, documents are the mainstay of data collection, so a systematic investigation of the early documents is hugely significant in setting up the data collection plan.

\section{Archival Records}

The investigator will depend on secondary data that are available in a variety of sources. Vogt et al. (2012) highlighted some of those resources, which can be used in this research. First of all, textual materials which have been published; these include books, academic journals, newspapers, and magazines, especially governmental official documents such as the urban morphology studies which have been done on the historical district (Vogt et al., 2012; Yin, 2009). Finally, some types of archival data can be accessed by using the internet (e.g. blogs and web pages) (Vogt et al., 2012). The research basically depends on archival data sources when studying the historical district as well as the transition areas at Jeddah; these include scholarly literature, newspaper articles, observations, architectural drawings, maps, blogs and oral histories (Groat and David, 2013; Yin, 2009; Vogt et al., 2012). There are certain benefits behind using the last technique, which is helpful in terms of acquiring data that have either been not documented, lost, or difficult to document" (Della Porta, 2014). Several keywords will be used as a criterion for selecting articles, e.g. King Abdullah Economic City, KAEC, Rabigh, and so on. Al-Watan, Al Eqtusadiah, and Makkah newspapers were among the most significant sources of data as they have reported heavily on the KAEC.

\section{Interview}

Some early literature showed that some people prefer interviews when seeking information of a personal nature, as it allows them to help interviewees concentrate on the main purpose of the interview (Albarqawi, 2012b). Yin mentioned that the interview is considered as a significant source of evidence for the case study research, because the majority of the case study research discuss either human issues or behavioral events (Yin, 2009; Yin, 2011). There are several types of interview in the case study design; however, this research will conduct In-depth and focus group interviews.

\section{In-depth Interview}

The researcher aims to conduct a variety of in-depth interviews that involve open-ended questions, thereby allowing respondents the opportunity to freely talk with residents, academic staff, and stakeholders such as practitioners, representatives of both the municipality of Jeddah and EMMAR Properties. Those interviews will take place during the data collection stage, while the average time for each interview should be around two hours (Yin, 2009; Yin, 2011). An investigator should consider two main aspects: focusing on commit- 
ment to the investigation plan, which is shown in the case study protocol, and secondly the investigator should not be asking biased questions. Yin mentioned that interviews in the case study design should be operated on two main levels; first, providing precise evidence which should help in answering the research questions (Yin, 2009). Second, at the in-depth level, the one significant task for the investigator is to ensure the safety of the interviewees which include not asking threatening questions (Yin, 2009). As a result, the investigator should aim to develop a state of rapport with respondents before conducting the interview in order to solve any lingering concerns by the respondents (Harbi, 1989; Yin, 2011). The interview form is written in English, and then translated into Arabic in case there are any difficulties in understanding English terminology, and the interviewee can choose to have the interview in either Arabic or English; for privacy reasons, the interviewee has full right to anonymity" (Della Porta, 2014).

\section{Interview Protocol}

The interview protocol includes several aspects such as writing the date and time of the interview, defining the place of the interview, the name of the interviewee, and any instructions that should be explained by the interviewer prior to conducting the interview. Those instructions include introducing the research topic and its aims to the interviewee, giving some information about the investigator, while the interviewee will be allowed to carefully read the consent form and ask any questions before signing the form. Recording device, note book for taking notes, and question sheet will be needed. Creswell (2013) mentioned that having an interview protocol is a useful way to recall the information from the interview by listening to the recording; he also encourages the researcher to construct a protocol for asking the questions during the interview (Creswell, 2013).

\section{Sampling}

The term "sampling" is widely used in the research field, which basically indicates choosing the sample(s) of the study by the researcher, such as the number of participants to be interviewed by the researcher. However, Yin (2011) has expanded that idea by dividing the samples of the study into two main levels: broader and narrower (Yin, 2011). Yin (2011, 88) also mentioned that the challenge for the researcher is how to define units of the study and giving reasons for this choice, and what is the number of participants in each unit. As a matter of fact, in this research the selected samples of the study are residential neighborhoods that include housing units in both the city of Jeddah and KAEC, which would represent a broader sample, while residents who live in the two cities mentioned earlier represent the narrower sample. In fact, there is no specific formula in the early literature which define the sampling size. Yin (2011) mentioned that "there is no formula for defining the desired number of instances for each broader and narrower unit of data collection in qualitative study. In general, larger numbers can be better that smaller numbers because a larger number can create greater confidence in a study's findings" (Yin, 2011). However, Creswell (2013) mentioned another practical approach in this respect, which is saturation. This simply means that the researcher should stop the process of collecting information when it is not offering any new knowledge or information (Creswell, 2013). Given the views of both Yin and Creswell, I decided to interview thirty people from those two cities (Jeddah and KAEC). The breakdown of this number is twenty residents, five academic staff, and five stakeholders that include practitioners, representatives of both the municipality of Jeddah and EMMAR Properties. However, more participants can join the study if saturation is not achieved. To conclude, Yin and Creswell assert the necessity of purposefully selecting participants to ensure that the researcher is able to collect the re- 
quired information from participants who have the background on the research topic, as they can provide useful data for the researcher (Yin, 2011; Creswell, 2013). This encouraged the investigator to select participants who have some knowledge on urban planning and designing cities from a sociocultural perspective.

\section{Focus Group}

Focus group is the second type of interviews in the case study research design, and it will be used to support and verify the outcomes of the interviews. Yin mentioned that the average time for conducting the focus group interview is around one hour (Yin, 2009). Early literature do not define the exact number of participants in focus group interview. However, Creswell (2013) mentioned that the researcher is expected to conduct an interview by engaging six to eight participants in each single group (Creswell, 2013). In this study, two focus groups will be enough, while the first one will be conducted with six residents who are living in KAEC, and the second one will involve six selected practitioners; both of these two groups will receive the same materials and they will be asked the same tasks; responses from those groups will be analysed in view of the research objectives (Yin, 2009; Creswell, 2013). The selected participants in the focus group interview are supposed to come from different sociocultural backgrounds. In addition, participants in the focus group interview should be selected in a purposeful way so as to serve the research aims and objectives. The main objectives of the focus group interview are to:

1. Identify the people's perception of the built environment at KAEC.

2. Define whether the neighborhood pattern as well as the spatial contexts of the housing units are appropriate for residents from different sociocultural backgrounds.

3. Show them different prototypes of the housing units in order to get their feedback on them, and then ask them to draw modifications on the floor plans so as to develop prototypes which match as much as possible their different sociocultural values.

\section{Site Observation}

The site observation process basically aims to collect information on the physical environment of the neighborhood as well as the sociocultural activities, which could help the investigator in examining patterns of sociocultural values when relating them to the neighborhood's urban fabric, housing design, and the built environment generally (Yin, 2009; Hancock and Bob, 2011). Moreover, this process will help the researcher in investigating the relationship between architectural features of houses' facades and the urban spaces of the neighborhood. One significant advantage for that process is that the presence of the house owner is not required to complete the investigation (Harbi, 1989). During site observation, taking field notes could offer great opportunity to register unexpected aspects such as:

-Residents' activities in the neighborhood and how they engage with the urban spaces, which may give an impression of different sociocultural backgrounds as in the utilization of open spaces in the neighborhood.

-Modifications of residential units, which have been carried out by residents due to sociocultural concerns.

However, there are two main types of site observation: direct and participant observations(Yin, 2009).

\section{Direct Observation}

The nature of the case study research, which required investigating the phenomenon within its natural context, ensures ample opportunities for conducting direct observations (Yin, 
2009). During the fieldwork, observations take different forms, which range from formal to informal observations (Yin, 2009). For example, formal observation includes official meetings, urban activities and the like; while with the informal ones, the investigator tries to develop a connection between different aspects in order to show a new aspect, e.g. the outline of the offices at the workplace could indicate the satisfaction of the employees on their physical environment in the workplace (Yin, 2009). To maximize the benefits of conducting the site observations, photographs should be taken for the studied phenomenon, which could help the investigator as well as any outside observer in understanding the phenomenon (Yin, 2009).

\section{Participant Observation}

investigators at this kind of observations are active observers who can participate in activities that take place during the site observation (Yin, 2009). For example, the observer can participate in sociocultural activities at the residential neighborhood to investigate or to identify how those activities have contributed to forming the urban elements. Yin (2009) has added other roles for the observers when studying the neighborhood (Yin, 2009). First, the observer can become a resident in the same neighborhood; second, the observer can participate in a functional role in the neighborhood such as being a book seller; third, finding a job at any local organization to become either a staff member or even decision maker (Yin, 2009). This kind of observation has been widely used in anthropological research, which involves investigating the sociocultural aspects of groups. There are many advantages for using direct observation. First, it ensures access to sociocultural activities, which involve different groups. Second, getting people's perceptions, especially those who are part of the case study. Third, ensuring great opportunities for the observer "to manipulate minor events" (Yin, 2009). However, there are some disadvantages, too. First, in this kind of observation, the observer loses the opportunity to work as an external observer(Yin, 2009). Second, due to being part of the event, the observer can become a proponent of the groups as a studied phenomenon (Yin, 2009). Third, this kind of observation requires too much time, attention, and skills (Yin, 2009). Fourth, this observation is based on the behavior of the groups, which could contribute either to conducting the site observations or creating difficulties acquiring the required evidence (Yin, 2009).

\section{Data Collection}

In order to expand the benefits of using the five sources of evidence, Yin mentioned three main principles that any investigator should take into account in order to ensure the validity and reliability of the evidence in a case study research (Yin, 2009). The first principle is using multiple sources of evidence, which provides great opportunities for the investigator to deeply investigate matters related to the history and behaviors of people (Yin, 2009). Yin (2009) has cited two main advantages for collecting evidence from multiple resources: "...the development of converging line of inquiry, a process of triangulation and corroboration..." (Yin, 2009). The second principle is creating a case study database, which focuses on developing a strategy for collecting, organizing, and documenting the collected evidence (Yin, 2009). There are four essential components to develop a database: notes, documents, tabular materials, and narratives (Yin, 2009). The third principle is developing a chain of evidence, which allows the external readers to follow up on the derivation process for the evidence, starting from the research questions, going through the different stages of study until reaching the conclusion, or starting from the conclusion and going 


\section{RESULTS AND DISCUSSION}

\section{Discussion}

From what was earlier mentioned about investigating people's perceptions of changes in their physical environment, I have derived a mode of inquiry in relation to two momentous periods in Saudi Arabia's history, namely: the modernization through oil and the National Transformation Plan Saudi Vision 2030. Advancing a new space of consciousness is the primary goal behind using this model in order to promote the evolution of Islamic civilization by investigating the sociocultural patterns and changes that will significantly bridge the gap between the imagined KAEC and its tangible presence (Albarqawi, 2012b).

Some early literature have cited that the research design should be developed after identifying the purpose of the study and the research questions; this step might help the investigator in evaluating the substance and aims of the study. The term Research Design, which includes a series of activities such as strategies, aims and plans, has been adopted to help address the research question (Dang and Sui Pheng, 2015; Sale et al., 2002). However, the researcher should follow two fundamental steps prior to setting up the research design: the researcher should clearly identify the substance to be studied, and the best approach towards answering the research questions and achieving the research aims (Babbie, 2013; Fageha, 2014).

Others have added to the aforementioned aspects other significant factors such as identifying the appropriate method to collect the data, sampling, and variables' interpretation (Cavana et al., 2001). The flowchart 1 below illustrates the different stages of the research which should help in achieving the study aims (Fig. 4). First of all, the research should begin by reviewing the related literature so as to expand the horizon of knowledge about the research topic and to identify the gap, which is not discussed in the early literature. The identified gap should be addressed when answering the research questions, which are divided into two parts: main questions and sub-questions. The literature review process should also help in developing a theoretical framework, thereby helping to form the interview questions. The research will use a case study design to obtain multifaceted responses which should help in answering the research questions.

The nature of the inquiry, which aims to explore how the concept of local identity is being expressed in contemporary urban development in Saudi Arabia with reference to the city of Jeddah and KAEC, has more or less determined the plan and methods of this research(Groat and David, 2013).

By applying this research method, the investigator can track the urban transformation in the city of Jeddah through analyzing and assessing the residential neighborhood patterns, housing design, and the built environment in order to generally understand the relationship between the city pattern and the sociocultural values and norms which make up the local Identity of this traditional city. As a matter of fact, tracking the urban transformation and sociocultural changes in different periods will help prove the basic Islamic determinants that contributed to forming the built environment at Jeddah, and to test how flexible those determinants are in providing an acceptable level of integration at the built environment in KAEC between Saudis and non-Saudis, especially Muslims and residents from other religions; they include urban pattern, housing design, and sociocultural values and norms. The outcomes should help stake holders and other researchers to better understand the basic Islamic determinants in terms of urban development which do not distinguish between people from different sociocultural backgrounds when it comes to forming the built environment, as they are mainly focused on achieving public benefits such as maintaining neighbors' rights. 
CONCLUSION

Shifting from oil dependency will greatly contribute to improving other aspects such as the economic situation, the urban scene, and so on. However, when launching new urban cities in Saudi Arabia, the focus should specifically be in easing the tensions between Saudis and Non-Saudis in terms of designing the housing units, residential neighborhoods, and the built environment generally, which significantly form the identity of residents, and ensuring they are compatible with their sociocultural values and norms. As an Islamic country, urban development in Saudi Arabia should express the country's local Identity but to keep away from the conservative approach by focusing on the Islamic determinants which should be respected when planning these new cities. Those basic Islamic determinants are focusing on the civic rights of residents by respecting the relationship between human beings and their built environment. So by assessing the story of urban development at Jeddah, the investigator can identify evidence on how people are interacting with their built environment which has been set up according to basic Islamic determinants, and how that interaction has changed through different periods as a result of depending on invented sociocultural values and norms due to the economic leap after the discovery of oil in 1938. As a result, the investigator can assess how flexible are the residential neighborhood patterns, housing design, and built environment generally at KAEC in providing an acceptable level of integration between Muslims and Non-Muslims, which include people from other religions and socio-cultural backgrounds.

LIMITATIONS AND RECOMMENDATIONS

This stuy has potential limitations. This study is limited in scope. ONly two cities from Saudi Arabia were focus of the study. Future research must include other cites as research subjects.

\section{REFERENCES}

Albarqawi, Wadia. 2012a. Urban transformation and architectural identity in Makkah. URL: https://goo.gl/5xJdpM (accessed March 16, 2017).

Albarqawi, Wadia. 2012b. Urban transformation and architectural identity in Makkah. Phd dissertation, Faculty of Architecture, Design and Planning, University of Sydney, Sydney, AU.

Al-Hathloul, Saleh, and Muhammad Aslam M. (1999). Creating identity in new communities: Case studies from Saudi Arabia. Landscape and Urban Planning 44, no. 4: 199-218.

Al-Naim, Mashary A. 2008. Identity in transitional context: Open-ended local architecture in Saudi Arabia. Archnet-IJAR, International Journal of Architectural Research 2, no. 2: 125-146.

Aly, Sherine SA. 2011. Modernization and regionalism: Approaches for sustainable revival of local urban identity. Procedia Engineering 21: 503-512.

Ayalp, N. 2012. Cultural identity and place identity in house environment: Traditional Turkish house interiors. Interior Architecture and Environmental Design Department, TOBB ETU University, Ankara, TR.

Babbie, Earl R. 2013. The basics of social research. Beimont, CA: Cengage Learning.

Baxter, Pamela, and Susan Jack. 2008. Qualitative case study methodology: Study design and implementation for novice researchers. The Qualitative Report 13, no. 4: 544-559.

Cavana, Robert Y., Brian L. Delahaye, and Uma Sekaran. 2001. Applied business research: Qualitative and quantitative methods. Queensland, AU. John Wiley \& Sons.

Christiansen, Bryan, Salih Yıldız, and Emel Yıldız. 2013. Modernization without Westernization: The Kingdom of Saudi Arabia. In Transcultural marketing for incremental and radical innovation. Hershey, PA: IGI Global. 
Dang, Giang, and Sui Pheng Low. 2015. Research Methodology Infrastructure Investments in Developing Economies: The Case of Vietnam. Singapore: Springer.

Della Porta, Donatella, ed. 2014. Methodological practices in social movement research. Oxford, UK: Oxford University Press.

Dul, Jan, and Tony Hak. 2008. Case study methodology in business research. New York, NY: Elsevier.

Elsheshtawy, Yasser. 2010. Little space, big space: Everyday Urbanism in Dubai. The Brown Journal of World Affairs 17, 53-71.

Elsheshtawy, Yasser. 2008. The evolving Arab city: Tradition, modernity and urban development. London, UK: Routledge.

Ernst \& Young. 2015. Economic cities-Opening vistas of growth in the Kingdom of Saudi Arabia.

Economic Cities Saudi Arabia. 2010. Economic Cities Saudi Arabia Opportunity Assessment for Cleantech Companies. Swiss Business Hub GCC, World Trade Center, Dubai, UAE.

Faden, Yousef MO. 1977. Urban Dwelling Environments: Jeddah, Saudi Arabia. Master dissertation, Massachusetts Institute Of Technolog, Massachusetts, MA.

Fageha, Mohammed K. 2014. Managing stakeholders' participation to improve project scope definition completeness and enhance project outcome. PhD dissertation, University of Melbourne, Melbourne, UK.

Glum, Julia. 2015. Saudi Arabia's youth unemployment problem among King Salman's many new challenges after Abdullah's Death. International Business Times.

Gharipour, Mohammad. 2015. Tradition versus modernity: The challenge of identity in contemporary Islamic architecture.

Groat, Linda, and David Wang. 2013. Architectural Research Methods. New York, NY: John Wiley and Sons.

Hancock, Dawson R., and Bob Algozzine. 2011. Doing case study research. A practical guide for begning researchesr. New York, NY: Teachers College Press.

Harbi, Thamer H. 1989. The Development of housing in Jeddah: Changes in built from the traditonal to the Modern. Unpublished PhD Dissertation, University of Newcastle, Newcastle, UK.

Howard, Ebenezer. 1946. Garden cities of to-Morrow. London, UK: Faber and Faber Ltd.

KEAC. 2013. King Abdullah Economic City KAEC Master Plan Planning Guidelines. Jeddah, SA.

Lahn, Glada and Paul Stevens. 2011. Burning oil to keep cool the hidden energy crisis in Saudi Arabia. London, UK: Chatham House.

Masad, Mohammad. 2008. Dubai what cosmopolitan city? ISIM Review 22: 10-11.

Moser, Sarah. 2012. Circulating visions of 'High Islam': the adoption of fantasy Middle Eastern architecture in constructing Malaysian national identity. Urban Studies 49, no. 13: 2913-2935.

Moser, Sarah, Marian Swain, and Mohammed H. Alkhabbaz. 2015. King Abdullah economic city: Engineering Saudi Arabia's post-oil future. Cities 45: 71-80.

Salagoor, Jamaludden Y. 1990. The influence of building regulations on Urban Dwelling in Jeddah. Faculty of Humanities and Social Sciences, Newcastle University, Newcastle upon Tyne, UK.

Sale, Joanna EM, Lynne H. Lohfeld, and Kevin Brazil. 2002. Revisiting the quantitative-qualitative debate: Implications for mixed-methods research. Quality and Quantity 36, no. 1: 43-53.

Saudi Commission for Tourism and National Heritage. 2013. Historic Jeddah, the Gate to Makkah. Report on the State of Conservation of the Property, Jeddah, Saudi Arabia.

Shoaib, Turki, and Ramin Keivani. 2015. Branding the new city: Exploring place branding in Saudi Arabia. Journal of Place Management and Development 8, no. 3: 254-265.

Stake, Robert E. 1995. The art of case study research. London, UK: Sage.

Vogt, W. Paul, Dianne C. Gardner, and Lynne M. Haeffele. 2012. When to use what research design. London, UK: Guilford Press.

Swiss Business Hub GCC. 2010. Economic cities Saudi Arabia opportunity assessment for cleantech companies. Dubai, UAE. 
Yin, Robert K. 2003. Case study research design and methods: Applied social research methods series, Los Angeles, CA: Sage.

Yin, Robert K. 2009. Case study research: Design and methods. New York, NY: Sage publications.

— This article does not have any appendix. - 